\title{
Performance Analysis of a Novel Decentralised MAC Protocol for Cognitive Radio Networks
}

\author{
Wajdi Alhakami, Ali Mansour and Ghazanfar A. Safdar \\ Department of Computer Science and Technology, University of Bedfordshire \\ Luton, LU1 3JU, United Kingdom
}

\begin{abstract}
Due to the demand of emerging Cognitive Radio (CR) technology to permits using the unused licensed spectrum parts by cognitive users (CUs) to provide opportunistic and efficient utilisation of the white spaces. This requires deploying a CR MAC with the required characteristics to coordinate the spectrum access among CUs. Therefore, this paper presents the design and implementation of a novel Medium Access Control (MAC) protocol for decentralised CRNs (MCRN). The protocol provides efficient utilisations of the unused licensed channels and enables CUs to exchange data successfully over licensed channels. This is based on the observation procedure of sensing the status of the Licensed Users (LUs) are ON or OFF over the licensed channels. The protocol is validated with the comparison procedure against two different benchmark protocols in terms of the network performance; communication time and throughput. Therefore, performance analysis demonstrated that the proposed MCRN perform better and achieve higher throughput and time benefits than the benchmarks protocols.
\end{abstract}

Keywords- Dynamic Spectrum Access; Common Control Channel; Cognitive Radio Networks; Cognitive Users; Licensed Users; Licensed Data Channels

\section{INTRODUCTION}

Spectrum is a natural resource, spanning a range from $3 \mathrm{KHz}$ to $300 \mathrm{GHz}$, while severely remains underutilised [1] [2]. In 1999, Joseph Mitola introduced a new technology called Cognitive Radio Network (CRN), which grants dynamic spectrum access (DSA) for the unused band. The technology permits the spectrum to be used more efficiently, by switching to the unused bands (white space). Thereby, it intelligently adapts its environment to facilitate transmission among the unlicensed cognitive users (CUs). Although CRNs admit the coexistence of both LUs and CUs in the spectrum, it restricts the communication process over a certain frequency by allowing CUs opportunistically access the available channel. However, protecting the LUs activities are required since those users have the priority to utilise the licensed channels. Thus, CUs are able to improve the spectrum utilisation through scanning and determining the white space before initiating the data transmission and vacating these occupied channels as soon as the LUs activities are detected.

The need for a dedicated common control channel (CCC) is essential in a cooperative approach of CRNs, since it plays a major role for guarantee the success of exchanging the control frames. It also facilitates to provide coordination and cooperation among CUs to proceed the process of sharing the spectrum sensing results and making the decision of selecting a licensed channel for data exchange between both senders and receivers [3] [4]. Moreover, although, the CCC is simple in its design [5], it easily overcomes the issues related to the allocation; establish the link between CUs and monitoring of a secure communication [6].

\section{COTRIBUtion}

The main contribution of this work is to improve the network efficiency and performance related to the communication time and throughput in a cooperative approach of decentralised CRNs. This is achieved by minimising the number of handshaking frames over the CCC between CUs which resulted in performing fast switching to the Selected Licensed Data Channel (SLDCHs) to initiate data exchange by those CUs. Thus, the following great advantageous will be delivered to both CUs and the entire network operation:

- Reserving the CCC for a shorter time to exchange the control information, as a consequence offering the network availability to a larger number of CUs

- Increasing the network's efficiency and performance by achieving higher throughput with less communication time

Thus, the paper is organised in 7 sections; section III briefly outlines the related work while the design and implementation of the proposed Medium Access Control (MAC) protocol for CRNs for data transmission among CUs is introduced in section IV. The performance evaluation of the proposed MAC protocol is presented in section $\mathrm{V}$ while the comparative analysis of the proposed and benchmarks protocols is discussed in section VI. The paper is concluded in section VII.

\section{RELATED WORK}

Several MAC protocols for decentralised CRNs have been conducted in the literature. These protocols aim to improve the throughput of the unused licensed channels. Their operations are different in terms of the channel access mechanism and the use of the CCC the licensed data channel selection criteria to exchange the control information. The authors in [7] proposed a MAC protocol for decentralised CRNs with improving the network throughput. The protocol does not consider a dedicated CCC instead it deploys a stable control channel for control information exchange among CUs. This is achieved when every $\mathrm{CU}$ maintains a status table of channels and indexes these channels frequently. Therefore, the channel which has the highest stability is determined to be the control channel for exchanging control frames between a pair of CUs. Another approach of MAC protocols uses CCC to facilitate the spectrum sharing process for exchanging the handshaking frames between two CUs is introduced in [8]. This leads to perform the initial synchronisation of the licensed channel over the CCC. However, this approach of allocating a CCC for CUs in a distributed CR environment is a challenge because of the 
absence of central entity, which provides the management part for determining the CCC channel, and the time difference of the spectrum resources. Therefore, there are two categories of MAC protocols consider the assumption of the existence of a single dedicated control channel which is available and reliable all the time for CUs to exchange their control information. The first type is used a licensed dedicated CCC to exchange the control information among cognitive CUs [9] [10] [11] [12] while the second category assume unlicensed dedicated CCC can be utilised by CUs to exchange their control information. Generally, the dedicated CCC assumption is commonly admitted for employments since it is a convenient place where all the CUs can launch and observe the ongoing packets of control information and efficiently simplify the architectures of the MAC protocols [13]. In [14], a MAC protocol called Cognitive Radio Enabled Multi-Channel MAC for CRNs is proposed. The protocol operates based on four handshaking frames over a dedicated control channel to exchange control information and solve the hidden node problem with considering a single transfer is equipped by each $\mathrm{CU}$ for both control and data channels. A dedicated and reliable CCC is assumed to be available all the time for the associated CUs to exchange their control information. The licensed data channel selection criteria is based on random selecting for transmitting data. When the LU appears (ON) to utilise the licensed channel, the CUs necessitates restarting the process of exchanging control information to switch to different available licensed channel for transmitting data. In [15], a Dynamic Spectrum Allocation MAC Protocol based on CR for QoS Support (DSA-MAC) is designed. The protocol is based on ZigBee channels with multiple transceivers assigned to each CU for accessing multiple channels simultaneously. Although the ZigBee channels have a range from 0-26, the proposed protocol specifies channel 0 as a dedicated CCC for adapting six 6 handshaking frames, whereas the rest of the channels (1 to 26) are used for transmitting data. If LU appears to use the licensed channels, CUs required to restart the process for selecting both CCC and data channel. The authors of [16] designed a MAC protocol called HC-MAC for CRNs in which a control channel is considered to exchange three different pairs of control frames. The first group includes C-RTS and CCTS frames, which are designed for contending and reserving the control channel while the second group involves S-RTS and S-CTS, which aim to exchange the available channels for data transmission between the sender and receiver. Thus, as soon as the data channel is determined both CUs switch to that channel and initiate the data exchange process after that another two frames known as T-RTS and T-CTS are launched over the control channel after the successful data exchange to notify other CUs about the end of the communication process by the current pair of CUs. This would enable other CUs contend the control channel for exchanging C-RTS and C-CTS frames. The work reported in [17] introduced a cognitiveradio-based carrier sense medium access with collision avoidance (CR-CSMA/CA) MAC protocol for CRNs. The protocol uses the CSMA/CA technique to access the channels utilised by CUs, and can be applied in three different scenarios, where a single channel, multiple channels, and a realistic CCC are used to exchange data among CUs. However, only the realistic CCC scenario is considered and applicable to this work, since it has the same feature as the proposed MCRN protocol, wherein a CCC is adopted for the exchanging control information. Therefore, the CR-CSMA/CA operates based on three handshaking frames over the CCC named as PTS, RTS and CTS. The Prepare-To-Sense (PTS) frame aims to ask neighbouring nodes to keep quiet for the next duration. Then spectrum sensing takes place to detect the channels states and determine the available channels. However, Request-To-Send (RTS) and Clear-To-Send (CTS) aim to exchange control information and update the NAV of the CUs.

Thus, despite the existing MAC protocols discussed beforehand accomplish successful utilisation of the unused white spaces by CUs, the performance of CRN's time and throughput can be improved if the number of handshaking frames is minimised. Since most of these protocols exchange more than two control frames over the dedicated CCC.

\section{DESIGN AND IMPLEMENTATION OF THE PROPOSED MAC PROTOCOL FOR CRNS (MCRN)}

The proposed MCRN is designed for decentralised CRNs where a set of CUs attempts to exchange their control information and data. This is a challenge in ad-hoc networks because of the lack of an existing centralised entity that acts as a Base Station or Access Point to determine the channels availabilities. Therefore, the assumption of an existence of a dedicated CCC that is available and reliable all the time to CUs for exchanging their control information is made and CUs are reachable within the same range. In addition, since energy detection technique is used widely to detect signals and has low computational and implementation complexities because of no need for the prior LUs' information over the licensed channels and particular designs to detect spread spectrum signals [18], it is adopted in the MCRN to detect the LUs activities.

\section{A. Control and Data transmission phases in MCRN}

Figure 1 demonstrates the network scenario, which both a dedicated CCC and multiple licensed data channels are used by the MCRN. Each CU is equipped with a pair of transceivers; a single transceiver is used for observing the ongoing information and exchanging a pair of control frames, RTS and CTS (in context of CR), between CUs over a CCC while the second is used for exchanging data and ACK frames over the SLDCHs. The CCC is allocated to only CUs within the same range for exchanging control information related to the available channels not occupied by LUs such as Cannels 2 and 3 ( $\mathrm{CH} 2$ and $\mathrm{CH} 3$ ) while $\mathrm{CH} 1$ is excluded as a $\mathrm{LU}$ occupies it. This information is recognised as a Free Channels List (FCL) that were sensed and determined by the sender and the most reliable licensed channel (SLDCH) selected based on the highest available time by the receiver. Thus, each $\mathrm{CU}$ contends the CCC to send and receive both RTS and CTS frames that include FCL and SLDCH respectively (the details of the communication process for each pair of CUs is discussed in section 4 and shown in figure 3 ). Once each pair successfully exchanged the control information, they switch to the SLDCH for initiating data communication. Hens the CCC will be vacated by the first pair and granted for the next pair of CUs who win in the contention process to reserve the CCC. However, if the LU's activities are detected for utilising the SLDCH during data transmission by an associated sensor, both 
CUs require vacating the channel immediately and restarting the entire process.

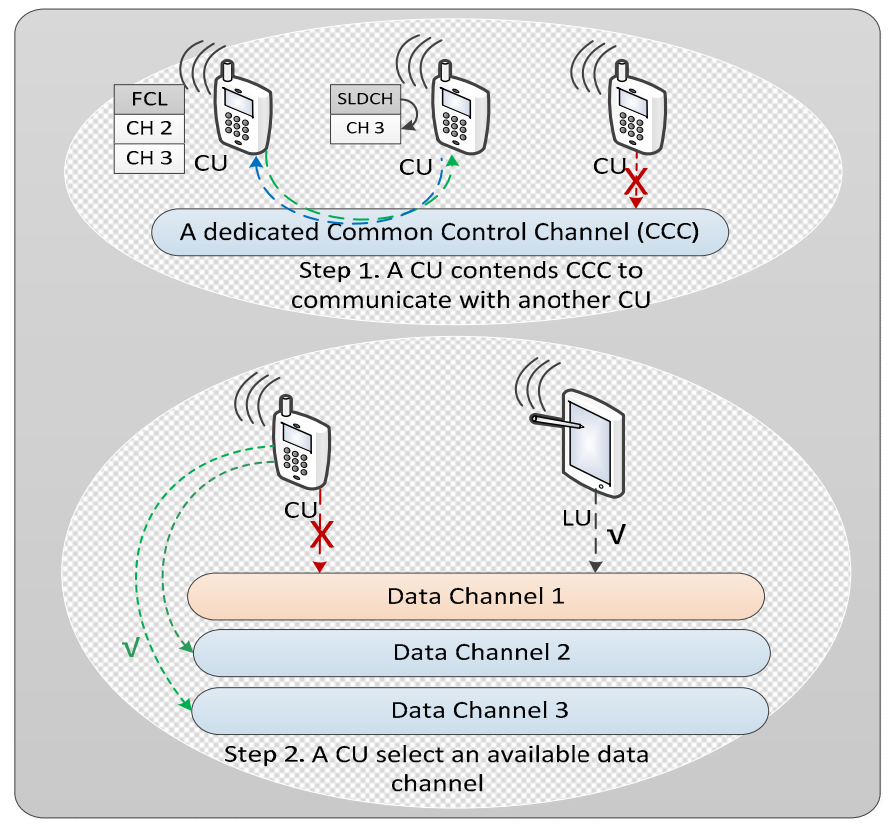

Figure 1: Network scenario

\section{B. Medium Access Control (MAC) for MCRN}

The DCF method offers the essential access and coordinates multiple CUs to utilise CCC to launch frame transmissions without the possibility of collision. It is based on the Carrier sense Multiple Access with Collision Avoidance (CSMA/CA) [19] [20] in which CUs require ensuring the CCC is clear for transmission before launching their control frames. This aims to avoid collisions and is achieved by applying channel access based on contention technique and random back-off time. If the channel is marked busy, then a CU needs to set random backoff time to avoid collision. Thus, in Figure 2, CU C needs to communicate with CU A. The process of the prior CCC access is based on the contention technique and $\mathrm{CU} \mathrm{C}$ requires checking the availability of the CCC for a time equals to DIFS before launching the RTS frames. If the CCC is busy then the $\mathrm{CU}$ applies random backoff time to seize the channel without any collisions occurring. Otherwise $\mathrm{CU} C$ wins access to the CCC and can launch the RTS frame because the channel is available for a period of time exceeding DIFS time. This leads to the neighbouring CUs, who are within the same range, can recognise the transmitted control frame and set their NAV for avoiding collisions that might occur. However, the intended destination (CU A) requires waiting time known as SIFS. Then, once the SIFS time has elapsed, CU A replies with CTS. Consequently, both CUs require switching to the next phase for initiating data transmission after waiting SIFS time.

\section{PERformance EVAluation of THE MCRN}

\section{A. The network parameters}

Since MATLAB simulator is extensively used for wireless technology research such as in [21] and [22], the proposed and the benchmark protocols are simulated in MATLAB for comparison, evaluation and validation purposes. As discussed in [23] a large portion of the TV white space becomes available for CUs to utilise.

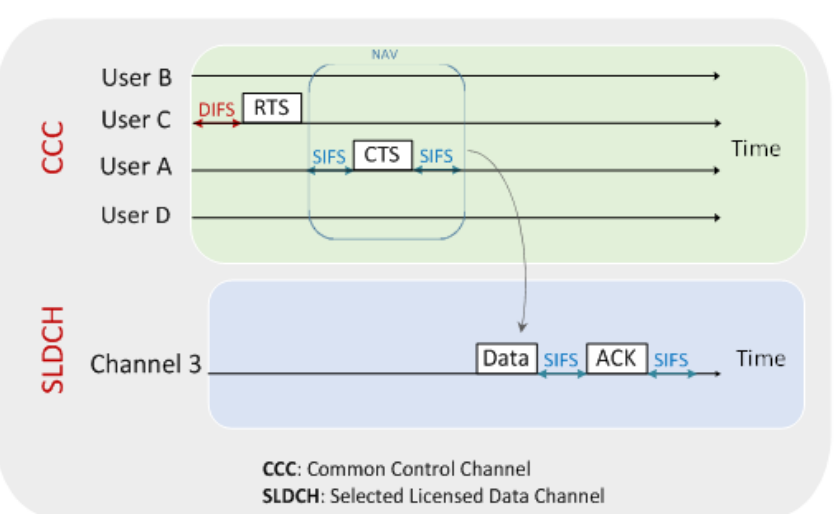

Figure 2: Timing and process of Medium Access Control (MAC) in MCRN

Thus, the TV portion; from 471.25 $\mathrm{MHz}$ to $607.25 \mathrm{MHz}$ is considered in the proposed and benchmark protocols for the CCC and 10 data channels to exchange both control information and data respectively. Therefore, several parameters are considered in the MCRN simulation to fulfill the entire communication process among 20 CUs. These involve a single $\mathrm{CCC}$ and 10 data channels used by CUs, considering the best data channel selection criteria for enabling the success of data transmission. Moreover, both CCC and Data channels have the same data rate of $11 \mathrm{Mbps}$ and the DSSS PHY layer characteristics are applied. In addition, 1500 bytes of data as a payload size is considered in the proposed scenario to analyse its influence on the entire communication over the data channels. Table 1 highlights the parameters used with their values in the MCRN.

\begin{tabular}{|c|c|c|}
\hline \multicolumn{3}{|c|}{ Table 1: The network parameters in MCRN } \\
\hline Parameters & Value & Description \\
\hline DIFS & $50 \mu$ secs & Distributed Interframe Space \\
\hline SIFS & $10 \mu$ secs & Short Interframe Space \\
\hline CCC-TR & $11 \mathrm{Mbps}$ & CCC Transmission rate \\
\hline DCH-TR & $11 \mathrm{Mbps}$ & DCH Transmission rate \\
\hline$N_{\mathrm{S}}$ & 2 & Number of Transmitters \\
\hline $\begin{array}{c}\text { PHY layer } \\
\text { Characteristics }\end{array}$ & DSSS & Direct Sequence Spread Spectrum \\
\hline RTS & 20 bytes & Request-To-Send \\
\hline CTS & 20 bytes & Clear-To-Send \\
\hline Data & 1520 bytes & Data frame \\
\hline ACK & 20 bytes & Acknowledgement frame \\
\hline $\mathrm{N}_{\text {CCC }}$ & 1 & Number of Common Control Channel \\
\hline $\mathrm{N}_{\text {SLDCH }}$ & 10 & Number of Data Channels \\
\hline $\mathrm{N}_{\mathrm{CUs}}$ & 20 & Number of CUs \\
\hline
\end{tabular}

\section{B. Communication time over control and data channels}

Figure 3 shows the communication time over the control and SLDCHs for $20 \mathrm{CUs}$. Each pair exchanges 1500 bytes of payload after the data channel is determined. Thus, the first pair (CU1 and CU2) accesses the CCC after the channel was sensed and determined to be available. Therefore, they necessitate $109.91 \mu \mathrm{secs}$ to successfully exchange the control information within the RTS and CTS frames over the CCC and $1132.91 \mu \mathrm{secs}$ for data transmission over the SLDCH. However, the second pair of CUs (CU9 and CU10) requires waiting time equals to $109.91 \mu$ secs to access the CCC which needs to be vacated by the first group of CUs. When the sender wins in the contention process for reserving the $\mathrm{CCC}$, $1242.82 \mu \mathrm{secs}$ is the required time to successfully complete the control information transmission, switching to the SLDCH 
and exchanging data between the sender and receiver over the SLDCH. The waiting time to launch the RTS frame belonging to Group 3 (CU11 and CU12) is doubled and equals to $2 * 109.91 \mu$ secs, since the control channel is busy for exchanging four control frames belonging to Groups 1 and 2 . In addition to this time, the third pair also requires $1242.82 \mu$ secs to complete their control and data exchange over both CCC and SLDCH respectively. The fourth group (CU3 and CU4) requires waiting time equals to $3 * 109.91 \mu$ secs to contend the CCC and then necessitate $1242.82 \mu$ secs to exchange the control information and data. This process remains the same for the next 6 pairs of CUs with the respect of adding the waiting time to start the contention and reserving the CCC for exchanging their RTS and CTS. Although, the data sizes are fixed and equal to 1500 bytes for
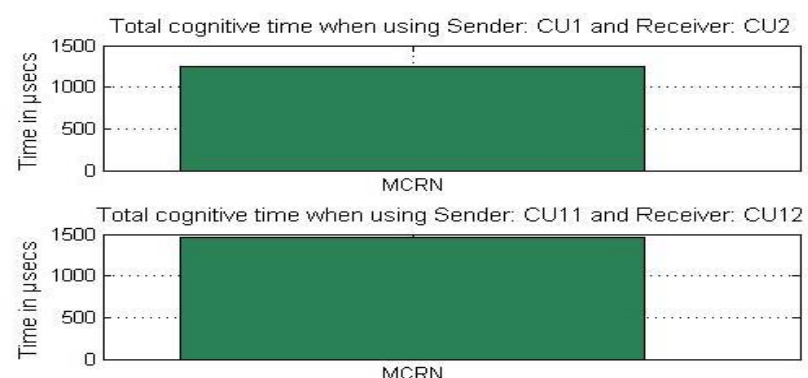

Total cognitive time when using Sender: SU13 and Receiver: SU14
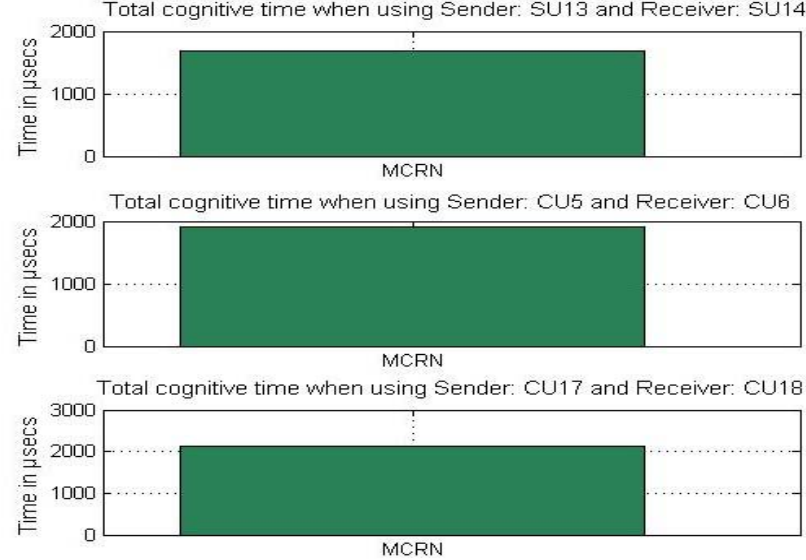

all groups and the same sizes of control frames, each pair of CUs requires $1242.82 \mu$ secs to successfully exchange the control information over the CCC and data over the SLDCHs. Therefore, the waiting times of group 5, group 6, group 7, group 8, group 9 and group 10 are $4 * 109.91 \mu \mathrm{secs}$, 5*109.91 $\mu$ secs, $\quad 6 * 109.91 \mu \mathrm{secs}, \quad 7 * 109.91 \mu \mathrm{secs}, \quad 8 * 109.91$ $\mu$ secs and $9 * 109.91 \mu$ secs in the same order. Consequently, the increase of the waiting time leads to the increase the communication time between CUs.

The total required time to exchange the control and data phases of the MCRN protocol (TMCRN) between senders and receivers successfully the following equation is applied.

$$
T_{M C R N}=\left\{T_{\text {DIFS }}+T_{R T S}+T_{C T S}+T_{\text {Data }}+T_{A C K}+3 * T_{\text {SIFS }}\right\}
$$
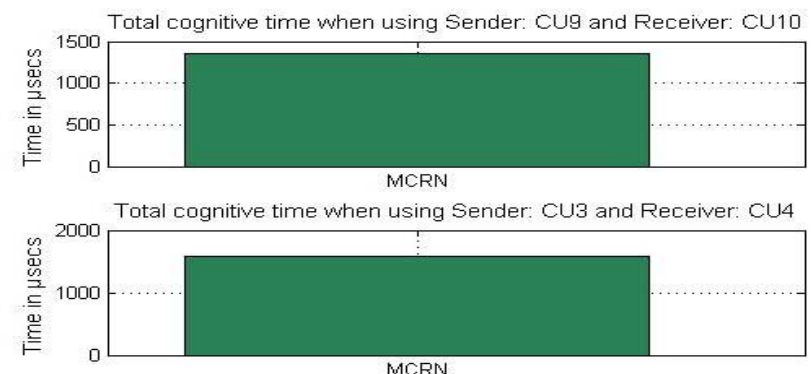

Total cognitive time when using Sender: CU19 and Receiver: CU20
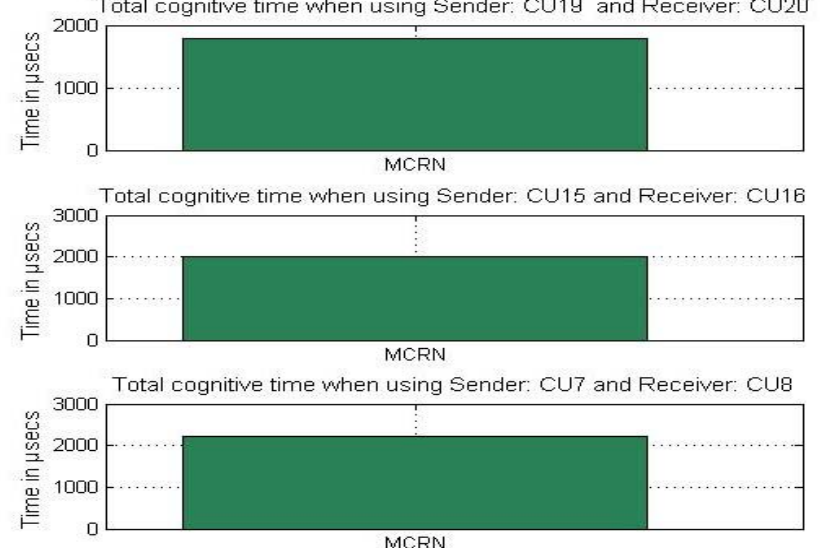

Figure 3: Total communication times for 10 pairs of CUs over control and data channels

However, Figure 4 demonstrates the communication activities of 10 pairs of CUs, and the prediction activities of LUs take place over 10 SLDCHs. In each data channel, the $\mathrm{x}$-axis explains which LUs are busy and which have free time for a period equals to $2 * 10^{4} \mu$ secs; the green areas show the busy signals for the LUs and the remainder are available to the CU. The red areas represent the CUs activities in the white space in each channel. However, the y-axis shows the amplitude of the LUs' and CUs' activities (the signal strength is represented by the ASCII format). As discussed in [22] CUs are allowed to share the spectrum with LUs with some restrictions such as the transmitted power's limitation. Thus 20 CUs are involved in the communication and initially two users contend the CCC and select a data channel that has the highest available time while the rest of CUs wait until the first group moved from the $\mathrm{CCC}$ to the SLDCH. Then, again contention process starts for the next pair wins the contention and so on. Generally, channel 1, which is occupied by the first group (CU1 and
CU2), has the maximum available time since LUs utilise the channel after a period of time equals to $1.8 * 10^{4} \mu$ ses. This makes this channel is the most reliable channel for the first pair of CUs to transmit data. In contrast, channel 10, which is occupied by the last pair (CU15 and CU16), has the lowest available time as the $\mathrm{LU}$ is predicted to appear in approximately $0.2 * 10^{4} \mu$ ses. This results in channel 10 having the lowest priority in terms of data channel selection criteria. Although, the time of the CU activities over these channels is equal, since they have the same data size (1500 bytes) to exchange, their communication is initiated at different times based on the waiting time of the control channel to be vacated and available for the next group of CUs. These waiting times are explained in details for each pair of CUs in figure 3. For instance, the first pair of CUs uses the CCC immediately after the successful contention process for the channel utilisation while the last pair (CU15 and CU16) had to wait

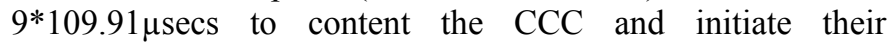


communication over CCC and SLDCH respectively. The time required over the $\mathrm{CCC}$ would influence the data channel

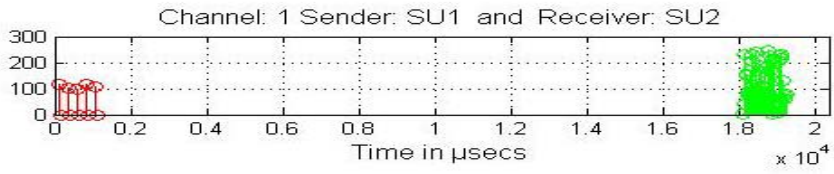

Channel: 3 Sender: SU3 and Receiver: SU4

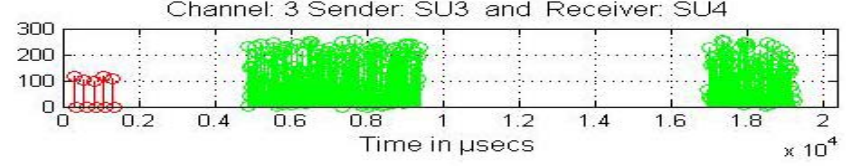

Channel: 5 Sender: SU13 and Receiver: SU14

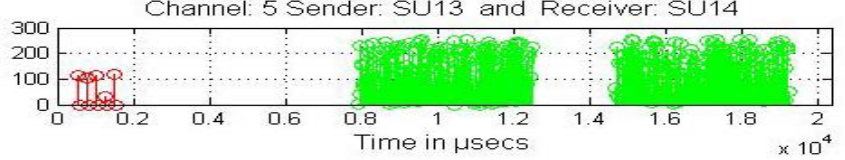

Channel: 7 Sender: SU7 and Receiver SU8

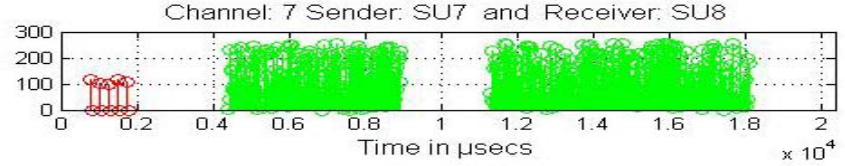

Channel: 9 Sender: SU19 and Receiver: SU20

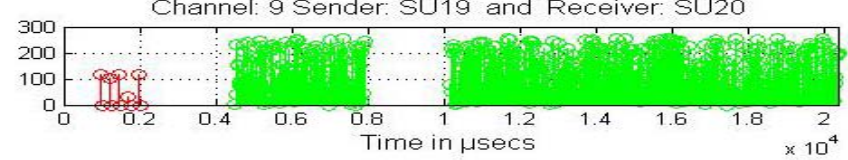

Figure 4: 1500 bytes of Data activities over the SLDCHs

\section{COMPARATIVE ANALYSIS OF THE PROPOSED AND BENCHMARKS PROTOCOLS}

Two different benchmarks protocols, recognised as Cognitive Radio-EnAbled Multi-channel MAC (CREAM-MAC) [14] and Cognitive-radio-based carrier sense medium access with collision avoidance (CR-CSMA/CA) [17] for CRNs, which were discussed in section II, are selected from among the available MAC protocols, since they are well known for decentralised CRNs, and are the closest to the proposed MCRN in the two networks features. These features include the use of a dedicated control channel in CREAM-MAC and a realistic $\mathrm{CCC}$ in $\mathrm{CR}-\mathrm{CSMA} / \mathrm{CA}$ to exchange control information among CUs over CCC, and multiple Licensed Data Channels (LDCHs), which are involved in data transmission. However, due to the long names of these protocols, they are renamed and abbreviated only in this paper, to CREAM and RACRN instead of CREAM-MAC and CRCSMA/CA respectively. Thus, from this point, these two abbreviations will be used and appear all the time when they are used. Both the communication time and throughput are the two network performance factors aiming for a comparative analysis of both MCRN against CREAM and RACRN.

\section{A. Handshaking frames over the control channel and data channels in MCRN, CREAM and RACRN}

Table 2 demonstrates the number of control and data frames, and their sizes in MCRN and the benchmark protocols. Despite all the protocols considering two different frames, known as data and ACK in the data phase, the number of control frames does not remain the same, since only 2 frames are used in MCRN, while 4 and 3 frames are exchanged in CREAM and RACRN respectively. This is considered as a clear contribution point of this research, since MCRN performs the negotiation with less handshaking frames, availability since the LUs have priority to utilise the licensed data channel at any time.
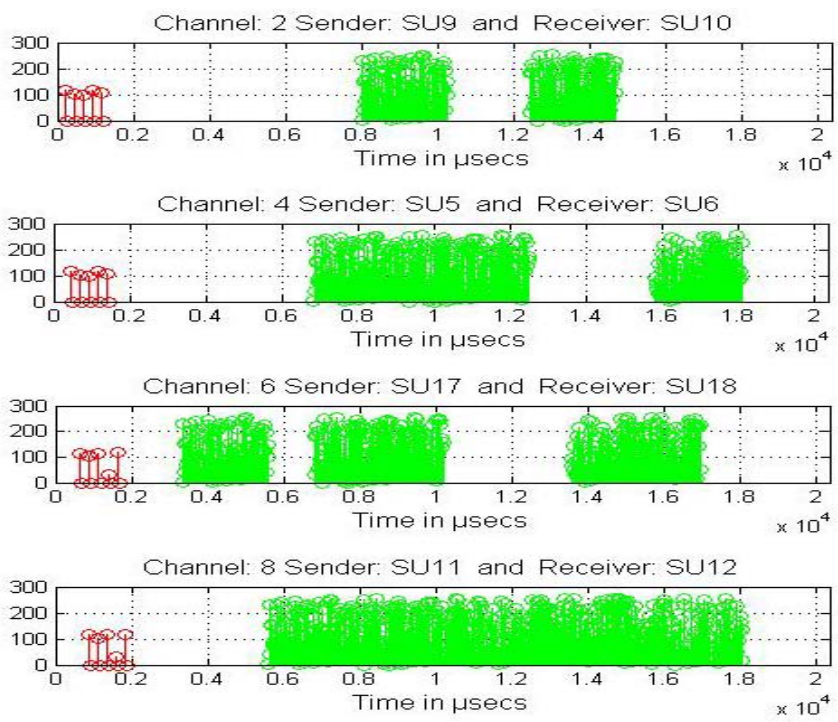

Channel: 10 Sender: SU15 and Receiver: SU16

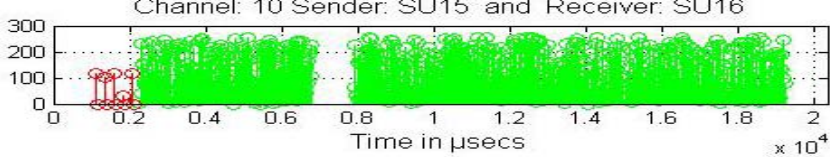

leading to a reduction of the communication time over the control channel and resulting in accomplishing fast switching to the SLDCH. Consequently, higher throughput is obtained by the MCRN compared to the benchmark protocols. Although, both CREAM and MCRN protocols use 20 bytes of control and data frames; whereas, RACRN uses different sizes and equals to 14 bytes in the PTS and CTS, while the RTS size remains the same, and equal to 20 bytes, this does not affect much in the communication time of the proposed protocol, since it reduces the number of the handshaking frames between CUs and resulting in achieving less time over the CCC and then led to perform fast switch to the SLDCH.

Table 2: Control and data frames in MCRN, CREAM and RACRN

\begin{tabular}{|c|c|c|c|c|}
\hline Protocols & $\begin{array}{c}\text { Control } \\
\text { frames }\end{array}$ & $\begin{array}{c}\text { Control frames' } \\
\text { sizes in bytes }\end{array}$ & $\begin{array}{c}\text { Data } \\
\text { frames }\end{array}$ & $\begin{array}{c}\text { Data and ACK } \\
\text { frames' sizes in } \\
\text { bytes }\end{array}$ \\
\hline MCRN & 2 & $\begin{array}{c}\text { RTS }=20 \text { and } \\
\mathrm{CTS}=20\end{array}$ & 2 & $\begin{array}{c}\text { Data }=1520 \text { and } \\
\mathrm{ACK}=20\end{array}$ \\
\hline CREAM & 4 & $\begin{array}{c}\mathrm{RTS}=20, \mathrm{CTS}= \\
20, \mathrm{CST}=20 \text { and } \\
\mathrm{CSR}=20\end{array}$ & 2 & $\begin{array}{c}\text { Data }=1520 \text { and } \\
\mathrm{ACK}=20\end{array}$ \\
\hline RACRN & 3 & $\begin{array}{c}\mathrm{PTS}=14, \mathrm{RTS}= \\
20 \text { and CTS }=14\end{array}$ & 2 & $\begin{array}{c}\text { Data }=1514 \text { and } \\
\mathrm{ACK}=14\end{array}$ \\
\hline
\end{tabular}

B. Time performance analysis of MCRN, CREAM and $R A C R N$

Table 3 demonstrates the difference in the time taken for a single pair of CUs to communicate with each other over both the CCC and a SLDCH in MCRN, CREAM and RACRN protocols. It is evident that the MCRN has less time over the CCC compared to the benchmarks, since it has less handshaking frames, which are RTS and CTS. In contrast, the CREAM necessitates the highest time due to 4 control frames are transmitted between sender and receiver CUs to determine the SLDCH. Moreover, the RACRN requires 3 control frames that are launched over the CCC to achieve the same goal. Over the SLDCH, the RACRN requires less time required to 
transmit 1500 bytes compared to the others protocols as the header field sizes belong to the data phase frames are less compared to those in MCRN and CREAM. Thus the total time of the frames' exchange in MCRN is $1231.18 \mu \mathrm{sec}, 1241.18$ $\mu$ sec in RACRN and $1286.09 \mu$ sec in CREAM. Although, the time is higher in MCRN compared to the RACRN by $1.64 \mu \mathrm{sec}$ for a single pair of CUs, MCRN performs better when more than a pair of CUs involved in the communication as will be discussed next. This because the communication time over the CCC is higher in RACRN compared to the MCRN, which achieves faster switching to SLDCHs.

Table 3: Time performance of the proposed and benchmark protocols

\begin{tabular}{|c|c|c|c|}
\hline Protocols & $\begin{array}{c}\text { Time over CCC } \\
\text { in } \mu \mathrm{sec}\end{array}$ & $\begin{array}{c}\text { Time over } \\
\text { SLDCH in } \mu \mathrm{sec}\end{array}$ & $\begin{array}{c}\text { Total time in } \\
\mu \mathrm{sec}\end{array}$ \\
\hline MCRN & 109.91 & 1132.91 & 1242.82 \\
\hline RACRN & 119.91 & 1121.27 & 1241.18 \\
\hline CREAM & 153.18 & 1132.91 & 1286.09 \\
\hline
\end{tabular}

Figure 5 illustrates the time spent on the communication process for five runs, including $4,8,12,16$ and $20 \mathrm{CUs}$ in the MCRN and benchmark protocols. This time refers to periods consumed over both the CCC to exchange the control frames, and the SLDCH to transmit 1500 bytes of data. Both the number and sizes of the handshaking control frames considerably affects the communication time for the frames' exchanges between senders and receivers. Consequently, it is clear that CREAM requires more time to exchange messages successfully than RACRN and the proposed MCRN protocol in each run. This is because there are 4 handshaking frames belonging to each pair of CUs, which are transmitted over the CCC in CREAM, while 3 and 2 frames for RACRN and MCRN respectively are launched over the same channel. Moreover, the overall times in the benchmarks and the MCRN increase as soon as the participating number of CUs is increased in each run. This is logical when each $\mathrm{CU}$ necessitates contention access the CCC to launch their RTS frames for data channel selection. However, a notable performance is achieved by the MCRN compared to the benchmarks, especially once the number of contributed CUs is increased this is due to the fewer handshaking frames that have been transmitted over the $\mathrm{CCC}$ and lead to fast switching to the SLDCH for data transmission. Although, the RACRN operates based on 3 handshaking frames over the $\mathrm{CCC}$, it is closer to the proposed MCRN than CREAM protocols in each run, due to the smaller sizes of the control frames transmitted, compared to those in the MCRN.

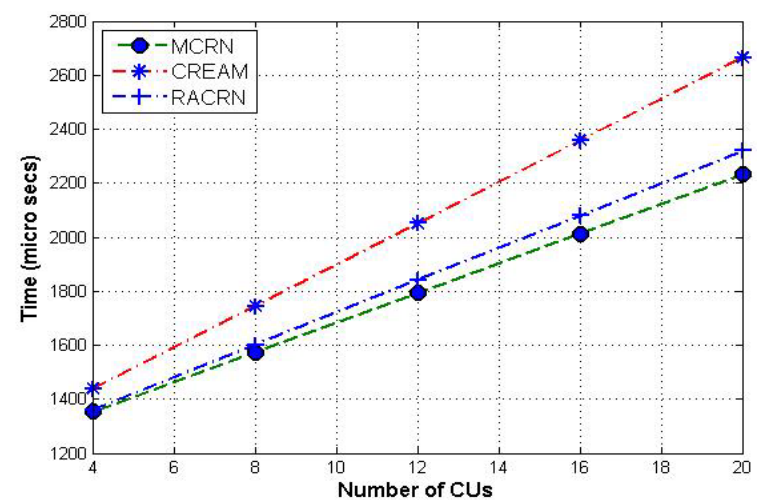

Figure 5: Communication time for 20 pair of CUs in MCRN, CREAM and RACRN
Thus, in the first run, where 2 pairs of CUs communicate, the total time required to successfully complete the communication process is $1439.27 \mu$ secs in CREAM, 1361.09 $\mu$ secs in RACRN and $1352.73 \mu$ secs in MCRN. However, in the second run, where 4 pairs of CUs are communicating, the total time for completing the data transmissions over the different SLDCHs increases by $306.09 \mu$ secs in CREAM, $239.82 \mu$ secs in RACRN and $219.82 \mu$ secs in MCRN. These increases resulted from exchanging the control frames, belonging to the $3^{\text {rd }}$ and $4^{\text {th }}$ participating pairs of CUs. The difference increase in the time of the benchmark protocols is significant compared to the MCRN when the number of participating CUs increased as shown clearly in the remaining runs. This due to the higher numbers of the control frames in the CREAM and RACRN compared to the MCRN and then require more time to be exchanged over a CCC.

\section{Throughput performance analysis of MCRN, CREAM and RACRN}

Figure 6 shows the throughput in both the MCRN and benchmark protocols for five runs, including 4, 8, 12, 16 and 20 CUs without LUs activities. The discussion of the throughput increase in the protocols for each run is associated with the number for the contributing CUs; since each sender transmits a message over a different SLDCH. Therefore, is apparent that the throughput increases dramatically in each run, since the SLDCHs are available to the CUs to initiate the data transmissions. However, although, the throughput in the MCRN is higher than the message delivery rate in the benchmarks for each run, the difference is significant between MCRN and CREAM. This is because the higher communication time is required among the contributing CUs to exchange the control frames over the control channel in the CREAM. Moreover, in each run of the MCRN and other benchmarks, the difference in throughput continuously increased, due to the increase in the number of the control and data frames exchange over the control and data channels respectively for each pair of CUs.

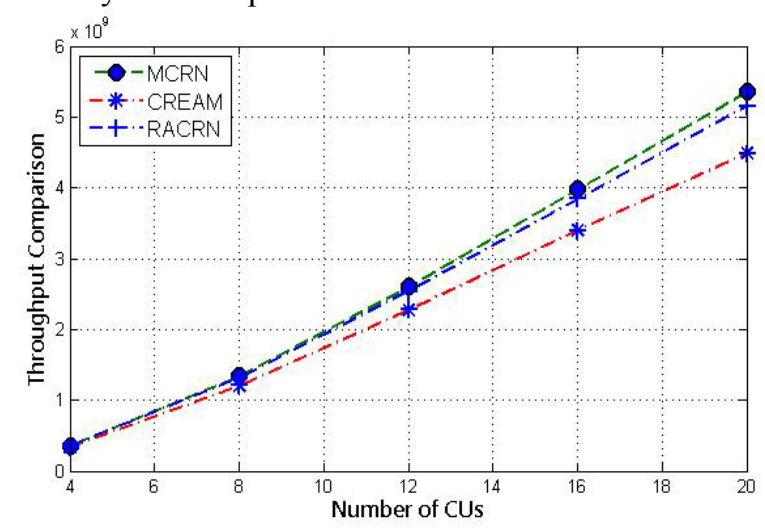

Figure 6: Throughput rate for 20 pair of CUs in MCRN, CREAM and RACRN without LUs activities

However, Figure 7 shows the message delivery rate in both the MCRN and benchmark protocols for five runs, including 4, 8, 12, 16 and 20 CUs with LUs activities. The same discussion of the throughput increases, and its comparison in the MCRN and benchmarks protocols in figure 6 is applied here. However, the LUs activities play a major role in utilising the LDCH with higher priority; therefore, it can be observed that the increase in the throughput in the second run is slight 
compared to that in the others runs, as the LU turned ON in the current run, for utilising a single $\mathrm{LDCH}$, and this led to the channel being vacated by the CUs. As a result, the CUs are unable to transmit data over this busy channel causing a decrease in throughput, compared to the situation in which the channel is available and utilised by the CUs. However, the status of the throughput increases in the remaining runs is dramatic compared to those in the second run, since the LUs remain OFF during the communication process.

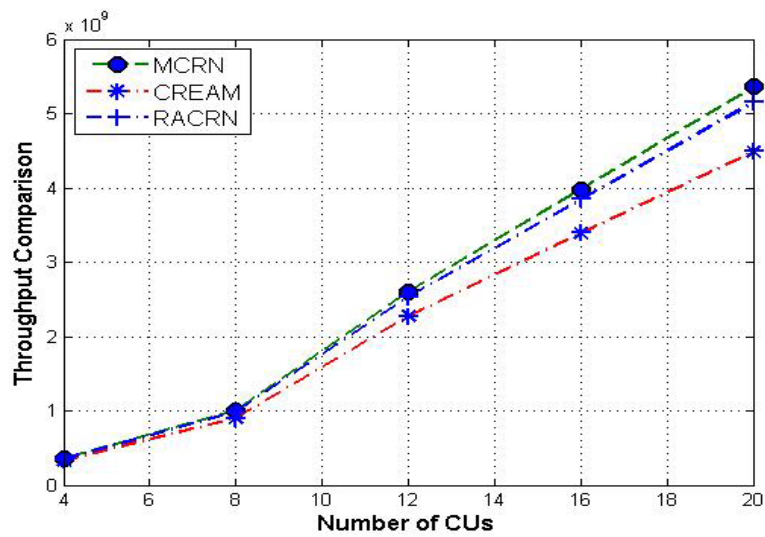

Figure 7: Throughput rate for 20 pair of CUs in MCRN, CREAM and RACRN with LUs activities

In general, the compassion results showed that the proposed MCRN achieved better performance compared to the benchmarks, since it aimed to reserve the CCC for a shorter time, due to exchanging fewer handshaking frames and made the CCC available for the next pair of CUs. This improved the communication time and data exchanges among the CUs, resulting in speeding up the switch to SLDCH to initiate data transmissions, which in turn led to achieve a higher throughput rate compared to the benchmark protocols.

\section{CONCLUSION}

MCRN protocol for distributed CRNs has been designed and simulated. The paper clearly identified the method of exchanging the control information among CUs over a dedicated control channel. Moreover, it provided the details of the main features of the MCRN protocol such as the number of the transceivers that associated to each $\mathrm{CU}$ to observe the ongoing activities over both the CCC and data channels which is selected and agreed based on the channel selection technique. Thus, a clear contribution of this research is accomplished by obtaining a higher throughput that is achieved by the MCRN compared to the benchmark protocols. This is due to the less communication time, which is inversely proportional with the throughput, is performed by the MCRN for a pair of CUs over CCC and subsequent fast switching to the SLDCH and initiate data transmission.

In future, a backup data channel will be considered in the implementation to improve the throughput of the unused white spaces. If the LUs activities are detected over the data channel, then CUs will be able to resume the communication over the backup data channel instead of repeating the entire process over the CCC. This effectively reduces the delay, which can be resulted from exchanging the RTS and CTS frames and contributes in achieving higher throughput.

\section{REFERENCES}

[1] L. Tang and J. Wu, "Research and analysis on Cognitive Radio Networks Security", Wireless Sensor Network, vol. 4, no. 4, pp. 120-126, 2012.
[2] T. Kamakaris, M. M. Buddhikot, and R. Iyer, "A case for coordinated dynamic spectrum access in cellular networks", in New Frontiers in Dynamic Spectrum Access Networks, 2005. DySPAN 2005. 2005 First IEEE International Symposium on, pp.289-298, 8-11 Nov., 2005.

[3] J. Jia, Q. Zhang and X. Shen, "HC-MAC: A Hardware-Constrained Cognitive MAC for Efficient Spectrum Management", IEEE Journal On Selected Areas In Communications, VOL. 26, NO. 1, vol. 26, no. 1, pp. 106 - 117, January 2008.

[4] Domenico, A. D., Strinati, E. C. \& Benedetto, M.-G. D., 2012. A Survey on MAC Strategies for Cognitive Radio Networks. IEEE Communications Surveys \& Tutorials, 14(1), pp. 21-44.

[5] Zhang, Y., Yu, G., Li, Q., Wang, H., Zhu, X., Wang, B., 2014. ChannelHopping-Based Communication Rendezvous in Cognitive Radio Networks. IEEE/ACM Transactions on networking, 22(3).

[6] Gavrilovska, L., Denkovski, D., Rakovic, V., Angjelichinoski, M., 2014. "Medium Access Control Protocols in Cognitive Radio Networks: Overview and General Classification," in Communications Surveys \& Tutorials, IEEE , vol.16, no.4, pp.2092-2124,

[7] G. P. Joshi, S. W. Kim and B.-S. Kim, "An Efficient MAC Protocol for Improving the Network Throughput for Cognitive Radio Networks", in Next Generation Mobile Applications, Services and Technologies, 2009. NGMAST '09. Third International Conference on,,pp. 271,275, 15. 2009.

[8] W. Alhakami, A. Mansour, and G.A.Safdar., "Spectrum Sharing Security and Attacks in CRNs: a Review", (IJACSA) International Journal of Advanced Computer Science and Applications, Vol. 5(1),.76-87, 2014.

[9] H. Su \& X. Zhang, "Cross-Layer Based Opportunistic MAC Protocols for QoS Provisionings Over Cognitive Radio Wireless Networks", IEEE Journal On Selected Areas In Communications, vol. 26, no. 1, pp. 118129, 2008.

[10] S.-J. Yoo, H. Nan, and T.-I. Hyon, "DCR-MAC: distributed cognitive radio MAC protocol for wireless ad hoc networks", Wireless Communications and Mobile Computing, vol. 9, no. 5, p. 631-653, 2009.

[11] R.-R. Chen, K. H. Teo,, and B. Farhang-Boroujeny, "Random access protocols for collaborative spectrum sensing in multi-band cognitive radio networks", IEEE Journal of Selected Topics In Signal Processing, vol. 5, no. 1, 124-136, 2011.

[12] S. Yin, D. Chen, Q. Zhang, and S. Li, "Prediction-based throughput optimization for dynamic spectrum access", IEEE Transactions On Vehicular Technology, vol. 60, no. 30, p. 1284-1289, 2011

[13] P. Ren, Y. Wang, Q. Du, and J. Xu, "A survey on dynamic spectrum access protocols for distributed cognitive wireless networks", EURASIP Journal on Wireless Communications and Networking, vol. 2012, no. 60, pp. 1687-1499, 2012

[14] X. Zhang and H. Su, "Cream-mac: cognitive radio-enabled multi-channel mac protocol over dynamic spectrum access networks", IEEE Journal Of Selected Topics In Signal Processing, vol. 5, no. 1, pp. 110-123, 2011.

[15] I. Joe and S. Son, "Dynamic spectrum allocation mac protocol based on cognitive", in Frontier of Computer Science and Technology, 2008. FCST '08. Japan-China Joint Workshop on, pp.24-29, 27-28 Dec. 2008.

[16] J. Jia, Q. Zhang and X. Shen, "HC-MAC: A Hardware-Constrained Cognitive MAC for Efficient Spectrum Management", IEEE Journal On Selected Areas In Communications, VOL. 26, NO. 1, vol. 26, no. 1, pp. 106 - 117, January 2008.

[17] Q. Chen; W. Wong; M, M.; Y. Liang, "MAC Protocol Design and Performance Analysis for Random Access Cognitive Radio Networks" Selected Areas in Communications, IEEE Journal on , vol.31, no.11, pp.2289,2300, November 2013

[18] Li, J., Feng, Z., Feng, Z. \& Zhang, P., A survey of security issues in Cognitive Radio Networks. In Communications, China, 12(3),132-150, 2015.

[19] N. Tadayon, and S. Aïssa, "Modeling and Analysis Framework forMultiInterface Multi-Channel Cognitive Radio Networks" IEEE Transactions On Wireless Communications, Vol. 14, NO. 2, 2015

[20] B. Dappuri and T. G. Venkatesh, "IEEE 802.11 DCF MAC Protocol for Cognitive Radio Networks: Cooperative Basic Access Vs RTS/CTS", in International Symposium on Communications and Information Technologies (ISCIT), 2014.

[21] Wei, W., \& Yong, Q. "Information Potential Fields Navigation in Wireless Ad-Hoc Sensor Networks". Sensors, 11(5), 4794-4807. 2011.

[22] Wei, W., Xiao-Lin, Y., Pei-Yi, S., \& Bin, Z. (2012). Holes Detection in Anisotropic Sensornets: TopologicalMethods. International Journal of Distributed Sensor Networks, Volume 2012, 1-9. 2012

[23] Maziar, N. Cognitive Radio Access to TV White Spaces: Spectrum Opportunities, Commercial Applications and Remaining Technology Challenges., in New Frontiers in Dynamic Spectrum, 2010 IEEE Symposium on ., pp.1-10, 6-9 April 2010. 